Published in final edited form as:

Best Pract Res Clin Haematol. 2007 March ; 20(1): 13-18.

\title{
The leukemic stem cell
}

\author{
Craig T. Jordan \\ James P. Wilmot Cancer Center, University of Rochester School of Medicine, Rochester, NY
}

\begin{abstract}
Malignant stem cells have recently been described as the source of several types of human cancer. These unique cell types are typically rare and possess properties that are distinct from most other tumor cells. The properties of leukemic stem cells indicate that current chemotherapy drugs will not be effective. The use of current cytotoxic agents is not effective in leukemia because the agents target both the leukemic and normal stem cell populations. Consequently, new strategies are required that specifically and preferentially target the malignant stem cell population, while sparing normal stem cells. Several well known agents are lethal for the leukemic stem cell in preclinical testing. They include parthenolide, commonly known as feverfew, and TDZD-8. They have undergone various levels of preclinical development, but have not been used in patients as yet in the cancer setting. These drugs and combinations of existing therapies that target the leukemic stem cell population may provide a cure in this disease. This article summarizes recent findings in the leukemic stem cell field and discusses new directions for therapy.
\end{abstract}

\section{Keywords}

leukemic stem cell; parthenolide; TDZD-8

\section{Introduction}

Approximately 50 years ago the concept was introduced that a stem cell may be connected to the origin and evolution of myeloid leukemia. To hypothesize the evolution of this disease, some form of normal stem or progenitor cell undergoes a mutation, giving rise to an entity that is functionally defined as a leukemic stem cell. The normal stem cells continue to differentiate into the hematopoietic lineage giving rise to erythrocytes, platelets, leukocytes, and granulocytes. The mutated stem cells have properties similar to the normal stem cells and can also differentiate into the hematopoietic lineage carrying the defect/s or can remain and accumulate as immature progenitor cells, also known as blast cells (Figure 1).

The chemotherapeutic agents used today effectively eradicate the blast cells in many patients. However, those same agents have very little if any activity at the level of the blast progenitor cell, the leukemic stem cell (LSC), which is biologically distinct from most of the cells that are found in a typical patient. An LSC is a functionally defined entity not necessarily named because it arises from a normal stem cell, but because it fulfills the same criteria used to define normal stem cells. These cells can undergo self renewal, are multipotent, and highly proliferative. The origin of the LSC has been the subject of considerable research in recent years. ${ }^{1}$

Craig T. Jordan, PhD, James P. Wilmot Cancer Center, University of Rochester School of Medicine, 601 Elmwood Ave, Box 703, Rochester, NY 14642, craig_jordan@urmc.rochester.edu, Ph: 001 585-275-6339, Fax: 001 708-575-3785

Publisher's Disclaimer: This is a PDF file of an unedited manuscript that has been accepted for publication. As a service to our customers we are providing this early version of the manuscript. The manuscript will undergo copyediting, typesetting, and review of the resulting proof before it is published in its final citable form. Please note that during the production process errors may be discovered which could affect the content, and all legal disclaimers that apply to the journal pertain. 
During normal developmental progression from stem cell to progenitor cell to mature cell, mutations may potentially occur at any point during this evolution, giving rise to a malignant entity. A mutation in a normal stem cell might give rise to a unit that could be considered an LSC. However, there is experimental evidence that suggests that mutations in a progenitor cell that no longer has all the characteristics of a stem cell can also give rise to an entity that can initiate and maintain leukemic disease. ${ }^{2}$ By extrapolation, it can be seen that more differentiated cells with the appropriate mutations may also give rise to the leukemic stem cell.

From a therapeutic perspective, the nature of the LSC may vary depending upon the stage during which it arose. Accordingly, drug resistance and various characteristics that are relevant to therapy may also differ, based on the origin of the diseased cell. Thus, it is possible to speculate that certain forms of leukemia that are relatively amenable to current therapies may derive from more differentiated cells that have certain intrinsic properties that are more readily addressed by conventional therapy. A plethora of new agents is available for treating leukemias, including kinase inhibitors, histone deacetylase inhibitors, cyclin D kinase inhibitors, heat shock protein inhibitors, methylation inhibitors, farnesyltransferase inhibitors, NF-kB inhibitors, and proteasome inhibitors. ${ }^{3,4}$ All of these agents affect specific mechanisms that may have gone awry. Despite the excitement and potential of all of these agents, there is not a single drug that has yet been validated as useful for eradicating the human LSC. An extremely important undertaking will be to fill this gap and to understand if and how all these new therapies are acting at the level of the stem cell.

Because anthracyclines, alkylating agents, nucleoside analogs, and topoisomerase inhibitors currently used in the treatment of acute myeloid leukemia (AML) often fail, they may not be targeting LSCs very effectively. ${ }^{5}$ In fact, there is no evidence that there is any selectivity or specific targeting of a leukemic versus normal stem cell by these agents. For example, Ara-C, which is a cycle-active agent commonly used in treating leukemia, shows virtually no activity with isolated LSCs. ${ }^{6}$ In contrast, this agent is effective when tested on blast cells from the same patient. There is a distinct difference in treating these two cell populations. When anthracyclines are tested in vitro, they are extremely cytotoxic both to LSCs and normal stem cells and there is no selectivity. However, there are ways that conventional therapies can be used in the appropriate combinations and appropriate methodologies, some of which have been previously published, to enhance the selective targeting and killing of leukemic stem cells.

\section{The leukemic stem cell}

LSCs can be isolated based on their cell surface markers using the currently available cellsorting technologies. Once the leukemic stem cells have been isolated, they can be analyzed using the same techniques applied in any type of cancer cell to understand the specific mutations and pathways that propel growth and survival. Tumor-associated properties in the LSC could include mutations in the kinase domains, transcription factors, and tumor suppressors, or alterations in the growth and survival mechanisms mediated through NF-kappa B (NF- $\mathrm{BB}$ ) or PI3 kinase, or changes in physiology, glucose metabolism, or responses to oxidative stress, to name a few. The results obtained from these analyses of LSCs can be compared to the unique properties of normal stem cells, which are relatively rare, have a quiescent cell cycle status, can efflux drug, and have self-renewal properties. Based on these findings we will be able to effectively develop regimens that will target LSCs. ${ }^{2}$

From this relatively broad perspective, it is difficult to choose the most effective pathways to target. A great deal of knowledge has been amassed on agents that target mitogenic tyrosine kinase mutations, such as the BCR/ABL mutation by imatinib mesylate, which induces apoptosis of primary chronic myeloid leukemia (CML) cells. ${ }^{7}$ Another example is the Flt3 
inhibitor CEP701, which induces apoptosis of primary AML blasts. ${ }^{8}$ However, there is no evidence that inhibiting these pathways is relevant to inhibiting the proliferating LSC.

As an alternative to inhibiting the activity of specific mutations in a cancer cell, it might be appropriate to consider the physiology of other unique mechanisms that maintain the viability and survival of these cells. Two such mechanisms have been suggested in the literature: the constitutive activation of the PI3 kinase pathway 9 and NF- $\mathrm{BB},{ }^{10}$ which are evident in LSCs. Inhibiting these two pathways might have therapeutic relevance. While no particular mutations or specific genetic events are associated with activation of the NF-кB or PI3-kinase pathways, converging events, such as multiple different mutations, may feed into these pathways.

However, such pathway modification may not be the only mechanism to produce leukemia.

Primitive human LSC populations can be selected by cell surface markers containing CD34+/ CD38-/CD123+ antigens. These cells are almost entirely quiescent, mimicking normal stem cells. As a result, cell cycle agents that are active in dividing cells will not be effective with this population. To characterize unique properties of LSCs, these purified populations have been examined using molecular analyses that look for activation of the NF- $\kappa \mathrm{B}$ pathway. As shown in Figure 2, activation of the NF- $\kappa \mathrm{B}$ pathway can be readily detected. Normal hematopoietic stem cells do not show activation of NF-kB. We believe that this is a leukemiaspecific phenomenon.

\section{New agents to target the leukemic stem cell}

Idarubicin with a proteasome inhibitor, parthenolide, and TDZD-8 are three examples of regimens that selectively target the leukemic stem cell. All three are able to inhibit NF- $\mathrm{kB}$ activity. The combination of idarubicin with various classes of proteasome inhibitors mediates selective apoptosis in LSCs while sparing normal cells. ${ }^{10}$ The second agent, parthenolide, is a naturally occurring sesquiterpene lactone ${ }^{11}$ that also selectively kills LSC populations with minimal activity in normal stem cells. Continuing studies with this agent in colony-forming assays have been used to measure progenitor cell function. This pattern of selectivity continues with the third agent, TDZD-8, which is lethal only to the leukemic population. Data on TDZD-8 is yet unpublished. From a preclinical standpoint, these types of molecules appear promising.

\section{Parthenolide}

Parthenolide is a small molecule that is the active compound in the plant known as feverfew, which has been used for centuries in herbal remedies to treat headache and inflammation. It is a potent inhibitor of NF- $\kappa$ B. Parthenolide selectively ablates myeloid leukemia cells at an $\mathrm{IC}_{50}$ of $2.5 \mu \mathrm{M}$ and causes apoptosis in primary human AML cells and blast crisis CML (bcCML) cells. AML progenitor and stem cells were analyzed in in vitro colony forming assays in a nonobese diabetic/severe combined immunodeficient (NOD/SCID) xenograft mouse model. Parthenolide preferentially targeted both AML progenitor and LSC populations. ${ }^{11}$ The only drawback is that parthenolide is not a good candidate for pharmacologic development because it is not particularly water soluble. We have collaborated with Peter A. Crooks, $\mathrm{PhD}$, at the University of Kentucky to develop analogs of parthenolide. The best candidate so far is a fumarate salt of dimethylaminoparthenolide. Its solubility in water is $\sim 1000$-fold greater than parthenolide and maintains its LSC-specific activity. It has been picked up by the NCI's RAID program and is currently undergoing further preclinical development. We hope to see this agent entering trials relatively soon.

TDZD-8

TDZD-8 (4-Benzyl-2-methyl-1,2,4-thiadiazolidine-3,5-dione) belongs to a family of molecules with an interesting history. A research group from the University of Madrid tested 
agents to inhibit the enzymatic activity of glycogen synthase kinase-3 beta (GSK-3 $\beta$ with the aim of treating neurologic diseases, such as Alzheimer's, in which the enzyme GSK-3 $\beta$ is very active. The agents they tested also have NF- $\mathrm{KB}$ inhibitory activity. One of these compounds, TDZD-8, is a non-ATP competitive inhibitor of GSK- $3 \beta\left(\mathrm{IC}_{50}=2 \mu \mathrm{M}\right)$, binds to the active site of GSK-3 $\beta$, and inhibits the activities of Cdk1/cyclin B, casein kinase-II, and protein kinase $\mathrm{A}$ and $\mathrm{C}\left(\mathrm{IC}_{50}>100 \mu \mathrm{M}\right)$. Preliminary data show that TDZD-8, similar to parthenolide, selectively induces the death of primary AML progenitor cells.

Further preliminary studies compared the efficacy of treating the AML progenitor cells with either parthenolide or TDZD-8. An overnight culture of AML progenitor cells expressing CD34+CD38- treated with either parthenolide or TDZD-8 showed that survival is rapidly impaired by TDZD-8 in comparison to parthenolide (half life of 120 minutes for cells in the presence of TDZD-8 vs > 360 minutes for those in the presence of parthenolide), relative to untreated controls. By contrast, there was very little to no activity in normal patient specimens.

In terms of the kinetics of these two agents, cells were treated with TDZD-8 or parthenolide at intervals of 30,60,120 ..1440 minutes and then assayed for viability. It became readily apparent between 6 and 12 hours that cells were dying in the presence of parthenolide. With TDZD-8, these cells were killed in as little as 30 minutes. When AML progenitor cells were introduced into NOD/SCID mice to rigorously assess stem cell potential, TDZD-8 inhibited the engraftment of AML leukemic stem cells, but did not significantly inhibit engraftment of normal hematopoietic stem cells. In a phenotypic analysis, cells were taken from the cell viability assay and introduced at each of the same time points into a functional assay such as progenitor cell colony-forming assays with striking results. The cells from the previous assay that were transferred into a functional assay had no detectable colony forming activity in as little as 30 minutes in the presence of TDZD-8. By comparison, parthenolide required an overnight time lapse before readily evident cell kill was observable. The mechanism of TDZD-8 is being investigated; however, from an empirical perspective, the extremely fast cell death with this agent is a very exciting development.

\section{Conclusion}

The two different agents discussed in this paper, parthenolide and TDZD-8, are chemically distinct entities. These compounds have the property of mediating cell death and inhibiting leukemic stem cell-specific activity. The empirical observation is that there are two types of stimuli required to bring about leukemia-specific cell death. The first is the inhibition of a survival pathway. The NF-kappa B and PI3 kinase pathways are affected by these drugs, but there could be others. Yet inhibiting pathways alone is not particularly toxic. However, when combined with a stimulus in the form of oxidative and/or genotoxic stress, the two signals together appear to be highly selective in killing leukemic stem cells, but are not particularly toxic at the normal stem cell level. Agents like parthenolide and TDZD-8 appear to simultaneously deliver both signals (Figure 3). A general criterion for selecting therapeutic regimens could be the ability of the agent to deliver both signals simultaneously.

In developing regimens that are more selective to leukemic stem cells, these agents should be developed for patient use and their activity should be validated at the level of stem cells. Similarly, when these regimens are used in patients, patient outcomes should be assessed in terms of the relative efficacy of targeting leukemic stem cells in vivo. The assays are currently available and can be applied to determine this.

\section{References}

1. Bonnet D, Dick JE. Human acute myeloid leukemia is organized as a hierarchy that originates from a primitive hematopoietic cell. Nat Med 1997;3:730-737. [PubMed: 9212098] 
2. Gilliland DG, Jordan CT, Felix CA. The molecular basis of leukemia. Hematology Am Soc Hematol Educ Program 2004:80-97. [PubMed: 15561678]

3. Tallman MS, Gilliland DG, Rowe JM. Drug therapy for acute myeloid leukemia. Blood 2005;106:1154-1163. [PubMed: 15870183]

4. Tallman MS. New strategies for the treatment of acute myeloid leukemia including antibodies and other novel agents. Hematology (Am Soc Hematol Educ Program) 2005:143-150. [PubMed: 16304372]

5. Kantarjian HM, Estey EH, Keating MA. New chemotherapeutic agents in acute myeloid leukemia. Leukemia 1996;10 (Suppl 1):S4-S6. [PubMed: 8618470]

6. Li TK, Houghton PJ, Desai SD, Daroui P, Liu AA, Hars ES, Ruchelman AL, LaVoie EJ, Liu LF. Characterization of ARC-111 as a novel topoisomerase I-targeting anticancer drug. Cancer Res 2003;63:8400-8407. [PubMed: 14679002]

7. Deininger MW, Druker BJ. Specific targeted therapy of chronic myelogenous leukemia with imatinib. Pharmacol Rev 2003;55:401-423. [PubMed: 12869662]

8. Smith BD, Levis M, Beran M, Giles F, Kantarjian H, Berg K, Murphy KM, Dauses T, Allebach J, Small D. Single-agent CEP-701, a novel FLT3 inhibitor, shows biologic and clinical activity in patients with relapsed or refractory acute myeloid leukemia. Blood 2004;103:3669-3676. [PubMed: 14726387]

9. Xu Q, Simpson SE, Scialla TJ, Bagg A, Carroll M. Survival of acute myeloid leukemia cells requires PI3 kinase activation. Blood 2003;102:972-980. [PubMed: 12702506]

10. Guzman ML, Swiderski CF, Howard DS, Grimes BA, Rossi RM, Szilvassy SJ, Jordan CT. Preferential induction of apoptosis for primary human leukemic stem cells. Proc Natl Acad Sci U S A 2002;99:16220-16225. [PubMed: 12451177]

11. Guzman ML, Rossi RM, Karnischky L, Li X, Peterson DR, Howard DS, Jordan CT. The sesquiterpene lactone parthenolide induces apoptosis of human acute myelogenous leukemia stem and progenitor cells. Blood 2005;105:4163-4169. [PubMed: 15687234] 


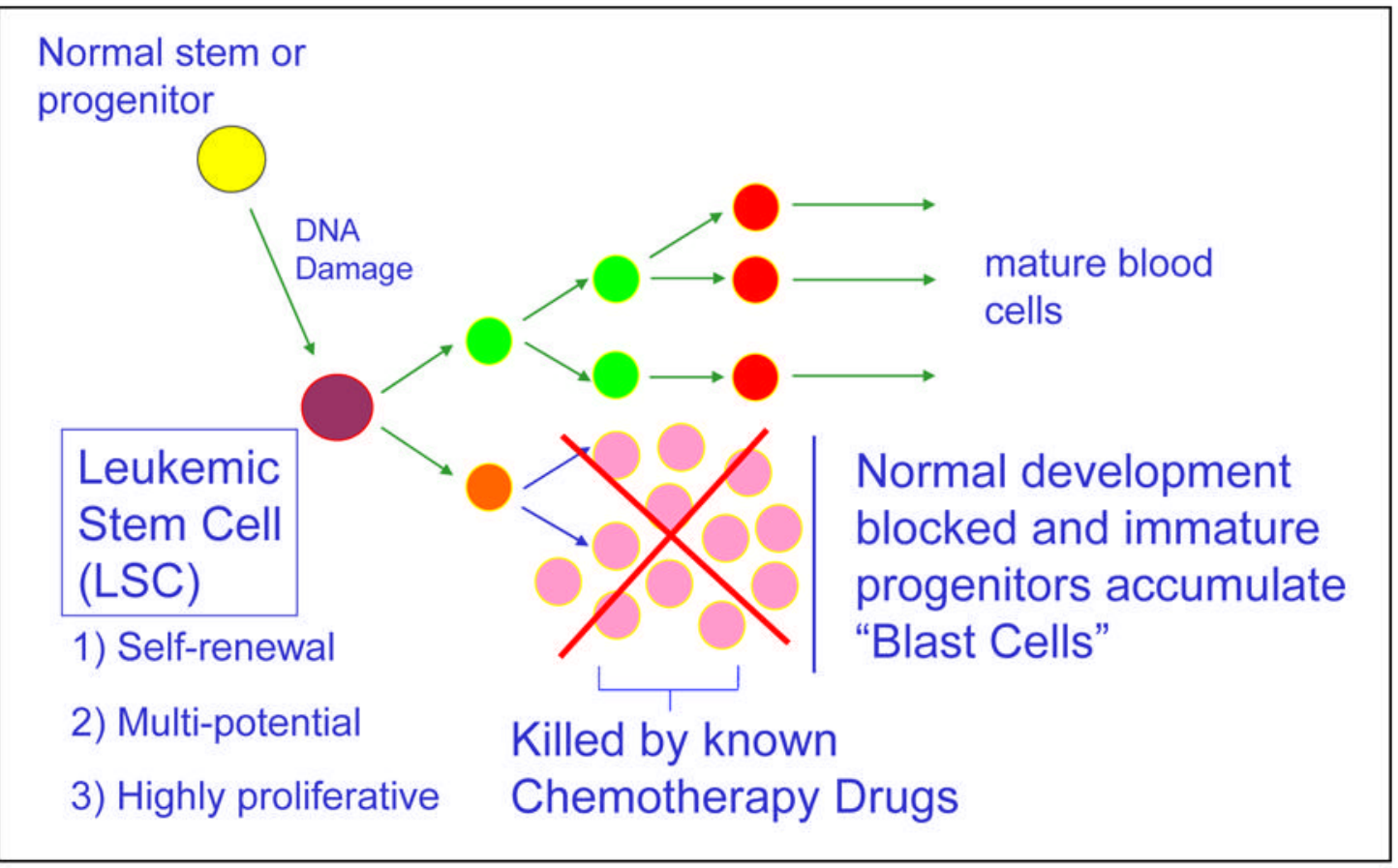

Figure 1.

Hypothesis of myeloid leukemia development

A normal stem or progenitor cell undergoes a mutation, giving rise to an entity that is functionally defined as a leukemic stem cell. The normal stem cells continue to differentiate into mature erythrocytes, platelets, leukocytes, and granulocytes. The mutated stem cells differentiate into the hematopoietic lineage carrying the defect/s or can remain as immature progenitor cells, also known as blast cells. 


\section{AML CD34+/CD38-/CD123+}
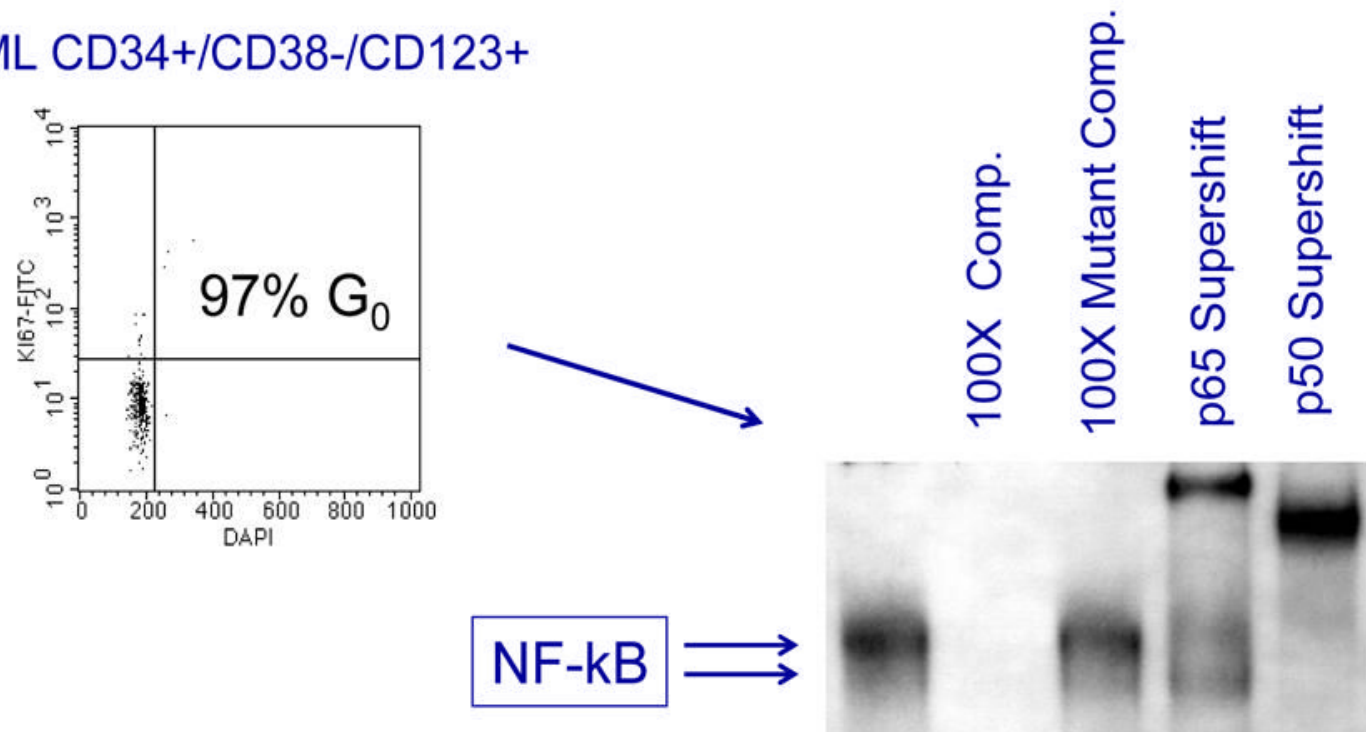

Figure 2.

NF-kB activity in primitive AML cells

Molecular analyses that readily show activation of the NF- $\kappa B$ pathway indicate the presence of leukemic stem cells. Normal hematopoietic stem cells do not show activation of NF- $\mathrm{kB}$. 


\section{1) Inhibition of survival factors (NF-KB/PI3K)}

- Proteasome inhibitors

- Parthenolide (analogs)

- TDZD-8

- ET-18- $\mathrm{OCH}_{3}$

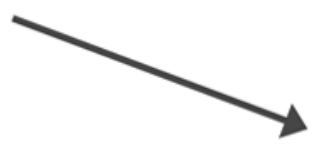

\section{2) Oxidative stress (DNA damage response)}

- IDR or other chemotherapeutics

- Parthenolide (analogs)

- TDZD-8

- Heat shock
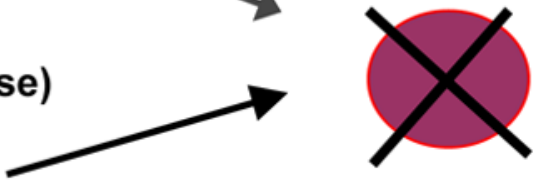

Figure 3.

Hypothesis to propel selective death in leukemic stem cells

Two types of stimuli are required to bring about LSC-specific cell death: inhibition of a survival pathway and oxidative or genotoxic stress. Parthenolide and TDZD-8 simultaneously deliver both signals. 